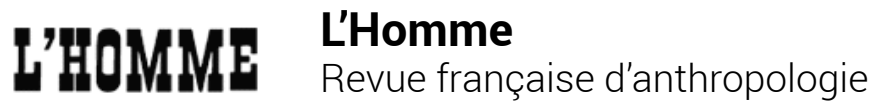

231-232| 2019

Cumulus : Hoarding, Hosting, Hospitality

\section{Sandrine Revet, Les Coulisses du monde des catastrophes "naturelles "}

\section{Bernard Formoso}

\section{Q OpenEdition \\ 1 Journals}

\section{Édition électronique}

URL : https://journals.openedition.org/lhomme/35846

DOI : 10.4000/lhomme.35846

ISSN : 1953-8103

Éditeur

Éditions de l'EHESS

\section{Édition imprimée}

Date de publication : 21 novembre 2019

Pagination : 309-311

ISBN : 978-2-7132-2794-3

ISSN : 0439-4216

Référence électronique

Bernard Formoso, «Sandrine Revet, Les Coulisses du monde des catastrophes «naturelles » ", L'Homme [En ligne], 231-232 | 2019, mis en ligne le 21 novembre 2019, consulté le 24 avril 2022. URL : http:// journals.openedition.org//homme/35846 ; DOI : https://doi.org/10.4000//homme.35846

Ce document a été généré automatiquement le 24 avril 2022.

(c) École des hautes études en sciences sociales 


\title{
Sandrine Revet, Les Coulisses du monde des catastrophes " naturelles "
}

\author{
Bernard Formoso
}

\section{RÉFÉRENCE}

Sandrine Revet, Les Coulisses du monde des catastrophes « naturelles ». Paris, Éd. de la MsH, 2018, 240 p., bibl., fig., annexes (« Le (Bien) commun »).

1 En ce début de $\mathrm{xxI}^{\mathrm{e}}$ siècle, les désastres causés par les accidents climatiques et l'activité géophysique ont acquis une saillance inédite du fait de leur fréquence, de leur fort impact humain et matériel, et de leur couverture médiatique, immédiate et massive. À la suite du cyclone de Bhola qui dévasta l'actuel Bangladesh en 1970, provoquant des dégâts restés sans équivalent, des organismes onusiens, mais également de nombreux autres opérateurs sont progressivement apparus. Les uns se sont spécialisés dans l'aide d'urgence, d'autres dans la réhabilitation des zones sinistrées ou bien dans la réduction des risques. Sandrine Revet retrace dans cet ouvrage l'enchaînement des événements, des initiatives individuelles et des processus collectifs ayant abouti à cet assemblage hétérogène d'intervenants. Elle analyse aussi les logiques et intérêts parfois divergents qui sous-tendent leurs activités en matière de sensibilisation des populations par le récit et l'image, de production de normes, ou encore de conception de dispositifs de prévention/information, d'interventions et d'appui à la résilience des victimes.

Bien que, placé sous l'égide d'instances de coordination onusiennes, dont la dernière en date est l'United Nations International Strategy for Disaster Reduction (UNISDR), un gouvernement international des catastrophes «naturelles » ait pris forme, le processus est loin d'être achevé. C'est la raison pour laquelle, et par emprunt à la sociologie interactionniste d'Anselm Strauss ${ }^{1}$, l'auteure préfère problématiser, en termes de «monde social » spécifique, les rapports unissant les intervenants du secteur, qu'ils soient bureaucrates, experts-techniciens, chercheurs, membres d'ONG, secouristes, représentants des assureurs ou vendeurs de systèmes GPS. Le concept de "monde 
social » est, selon elle, le mieux adapté pour rendre compte de la démarche prospective, tournée vers l'émission et la stabilisation de modes d'action efficaces, qui motive ces agents répartis en de multiples segments et dont l'articulation n'est pas lisible de prime abord. Ce monde, qui se construit en liaison avec ceux, plus anciennement constitués, de l'aide humanitaire et du développement, prend corps dans « des représentations, des langages, des temporalités, des lieux, des rêves, des outils, des normes, des kits et des procédures" qui sont cimentés par un alliage symbolique, en l'occurrence la réduction des risques de catastrophe (p.14). Reprenant une méthodologie initiée par Jean-Pierre Olivier de Sardan ${ }^{2}$, l'auteure explore ces différents registres avec l'étude de situations qui illustrent les interfaces entre les acteurs, les institutions dont ils se réclament et les systèmes de représentations qui les influencent. Entre 2009 et 2015, Sandrine Revet a observé concrètement les activités du bureau genevois de l'UNISDR et a notamment participé aux global platforms, ces grands forums mondiaux que l'instance organise tous les deux ans. Elle a également suivi via Internet et des entretiens téléphoniques, l'activité de différents acteurs, afin de s'imprégner de leur langage. Enfin, elle a assisté à des formations et à des exercices de simulation dans différents pays (Venezuela, Mexique, Pérou, Haïti et Japon).

Dans la première partie du livre, Sandrine Revet montre comment le monde des catastrophes "naturelles " s'est formé à partir d'organes de coordination créés par l'ONU, d'« entrepreneurs frontière » (à l'instar de l'ancien conseiller du président Jimmy Carter et séismologue, Frank Press) reliant plusieurs univers, et de récits qui portaient en eux un certain nombre d'oppositions structurantes. Parmi ces oppositions, l'une des plus importantes est celle qui démarque le monde des secours de celui de la prévention. Autant le premier agit dans l'urgence, son organisation est militaire et il applique des protocoles d'efficacité immédiate à forte connotation morale ("sauver le maximum de vies »), autant le second relève de l'analyse a posteriori, s'inscrit dans la longue durée, dans une agora politique et vise à responsabiliser les populations. Une autre divergence d'orientation significative sépare une approche aléa-centrée des catastrophes, qui privilégie les causes naturelles, d'une autre, influencée par la pensée marxiste, qui met l'accent sur les facteurs de vulnérabilité produits par les sociétés, qu'ils soient politiques, économiques ou sociaux. Le fait que, dans le titre et le texte de l'ouvrage, Sandrine Revet place systématiquement entre guillemets le qualificatif naturel associé à catastrophe, témoigne de sa volonté de ne pas prendre position entre ces deux modèles interprétatifs.

4 Prenant acte du rôle essentiel joué par les médias et du poids des images dans la sensibilisation du public, l'auteure consacre une section entière à l'iconographie produite par le monde des catastrophes «naturelles ». Elle souligne les préoccupations morales qui la sous-tendent: éviter la commisération et toute expression manifeste d'un paternalisme néocolonial. Cette approche met en avant des femmes et des enfants pour suggérer, selon un message teinté d'ambiguïté, leur vulnérabilité et leur besoin de protection, mais aussi leur faculté de résilience, portée en particulier par la jeune génération. Pour leur part, les hommes sont surtout présents dans l'évocation des moyens d'agir face aux catastrophes, en tant qu'acteurs de la prévention ou de la réhabilitation.

5 Contrairement aux pandémies ou au réchauffement climatique, les catastrophes « naturelles » touchent des populations localisées et, dans leur cas, l'aide internationale peut être contrariée par le souverainisme de certains États-nations. Pour contourner 
cette difficulté, les agences onusiennes et d'autres opérateurs ont progressivement élaboré des récits consistant à présenter ce type de désastres comme des crises complexes qui dénotent des risques globaux du fait de leur coût humain, des conflits et migrations qu'elles génèrent et de leur impact négatif sur la croissance économique mondiale. Dans la deuxième partie de l'ouvrage, Sandrine Revet montre la convergence des intérêts et les représentations qui étayent ces récits. Ceux-ci, à l'origine véhiculés par les grandes compagnies de réassurance, se nourrissent du supposé déclin de l'Étatnation et d'une notoire défiance des experts à l'encontre des gouvernements du Sud. Ils ont trouvé un surcroît de légitimité avec l'accroissement des risques nucléaires et autres dangers invisibles. L'accent est alors mis sur la prévention. Les notions clés de cette nouvelle doxa sont la menace all hazards donnée comme permanente et la " sécurité (humaine) globale » qui lui répond dans l'idéal. Des dispositifs d'alerte et de coordination, prenant appui sur les technologies séismologiques, géo-satellitaires et informationnelles les plus sophistiquées, confèrent une certaine capacité opérationnelle à cette gestion internationale des catastrophes. Celle-ci prend corps notamment dans le Global Disaster Alert and Coordination System. Créé conjointement par l'ONU et la Commission européenne en 2004, il a pour vocation d'anticiper les tremblements de terre, les tsunamis, les éruptions volcaniques, la trajectoire des ouragans et de lancer des alertes graduées en fonction des risques. Il facilite aussi la mise en place de structures centralisées de gestion des secours et de l'aide humanitaire.

6 L'internationalisation des catastrophes « naturelles » passe aussi par l'élaboration d'un système universel de normes en vue d'une gestion vertueuse des crises et d'une prévention efficace des risques. Le corps de savoirs et de modes d'action érigés en " désastrologie » dont participent ces normes, mais aussi les expériences partagées par les secouristes et autres acteurs au fil de leur côtoiement sur le terrain aboutissent à une communauté de pratiques et cultivent, chez ces intervenants, le sentiment qu'ils forment une grande famille. Sandrine Revet dresse l'inventaire critique des initiatives qui contribuent à la mutualisation des connaissances et à entretenir ce sentiment. Ce fut d'abord la création, en 1974, d'une base de données, le NatCatService, à partir des archives du réassureur allemand Munich Re, puis, en 1983, la constitution de l'Emergency Events Database par le Centre de recherches en épidémiologie des désastres de l'Université de Louvain-la-Neuve. Ce service recense les événements ayant causé au moins dix morts et entraîné une intervention internationale. Enfin, en réaction à ces initiatives qui prenaient peu en compte la vulnérabilité des sociétés, des chercheurs ont créé le site Desinventar, qui enregistre les petits et grands événements rapportés par les journaux locaux. Les informations collectées par ces différents dispositifs font l'objet d'une standardisation lexicale à partir d'un glossaire international établi par l'ONU en 1992, et servent à l'élaboration de modèles probabilistes des catastrophes.

7 Enfin, dans une troisième partie, Sandrine Revet se penche sur les stratégies de prévention et d'incitation à la résilience mises en œuvre par les acteurs du monde international des catastrophes "naturelles». Elle montre qu'afin de gagner la participation des populations aux exercices de simulation et de les sensibiliser aux systèmes d'alerte précoce (SAP), la rhétorique des experts accentue la probabilité des risques au point de faire des catastrophes une occurrence inévitable. Le problème, note-t-elle, est que les exercices de simulation reposent sur des simulacres ritualisés de remise en ordre, résultant d'une simplification outrancière de la complexité des 
désastres. La notion de résilience promue par les ONG et les organismes d'aide au développement fait, elle aussi, l'objet de controverses, tant elle repose sur le prédicat fictionnel d'un retour à la normale, lorsqu'elle ne sert pas d'outil d'ajustement low cost aux conditions changeantes imposées par le néolibéralisme économique.

Ce livre est une étude très documentée, qui procède d'une reconstitution historique et d'une analyse critique fines d'un champ d'intervention globalisé encore peu connu et pourtant en plein essor. Sa lecture intéressera tout autant les chercheurs qui travaillent sur les risques et les catastrophes «naturelles ", que ceux qui questionnent les limites des ingénieries de l'humanitaire et du développement.

\section{NOTES}

1. Cf. Anselm Strauss, La Trame de la négociation. Sociologie qualitative et interactionnisme. Éd. par Isabelle Baszanger, Paris, L'Harmattan, 1992 (« Logiques sociales»).

2. Cf. Jean-Pierre Olivier de Sardan, Anthropologie et développement. Essai en socioanthropologie du changement social, Paris, Karthala, 1995 (« Hommes et société »). 